dose between the two groups was not significant $(P=0.52)$. Interestingly, $15(73 \%)$ of the 19 women who gave birth to infants with malformations were taking multivitamin and folic acid supplementation at the time of conception.

Original article Holmes LB et al. (2008) Increased frequency of isolated cleft palate in infants exposed to lamotrigine during pregnancy. Neurology 70: 2152-2158

\section{Risk of stroke elevated by use of rofecoxib or valdecoxib}

Rofecoxib and valdecoxib have been withdrawn from the market because they increase the risk of cardiovascular events. To determine whether these drugs and other types of selective cyclooxygenase 2 inhibitors (coxibs) and noncoxib NSAIDs can also increase the risk of stroke, Roumie et al. conducted a retrospective study of 336,906 individuals (aged 50-84 years) enrolled in the Tennessee Medicaid program.

Over a follow-up of 989,826 person-years, 4,354 hospitalizations for stroke were recorded, $89.3 \%$ of which were ischemic. Among nonusers of NSAIDS (the reference group) there were 4.51 strokes per 1,000 personyears. By comparison, users of rofecoxib had an increased risk of incident stroke (adjusted hazard ratio $1.28,95 \% \mathrm{Cl} 1.06-1.53$ ), as did users of valdecoxib (adjusted hazard ratio $1.41,95 \% \mathrm{Cl} 1.04-1.91)$. None of the other NSAIDS examined (celecoxib, naproxen, ibuprofen, diclofenac and indomethacin) were associated with an increased risk of incident stroke. Restriction of the analyses to new users of NSAIDs produced similar results. Compared with the reference group, individuals who began rofecoxib having not received any other NSAID in the 365 days before filling their first rofecoxib prescription had a 1.46-fold increased risk of incident stroke, and new users of valdecoxib had a 1.39-fold increased risk.

These results indicate that the risk of stroke is increased with the use of either rofecoxib or valdecoxib. By contrast, none of the other NSAIDs examined seem to affect stroke risk. The authors advise caution when prescribing NSAIDs with rofecoxib-like or valdecoxib-like coxib selectivity.

Original article Roumie CL et al. (2008) Nonaspirin NSAIDS, cyclooxygenase 2 inhibitors, and the risk for stroke. Stroke 39: 2037-2045

\section{Delayed myelin destruction underlies late CNS damage after chemotherapy}

Although many studies have reported delayed CNS damage following systemic exposure to chemotherapy, little is known about the mechanisms underlying these late effects. Now, Han et al. have developed an animal model of chemotherapy-induced damage to the CNS and have demonstrated that systemic treatment with a single chemotherapeutic agent, 5-fluoruracil (5-FU), is sufficient to cause delayed CNS damage in mice.

Initial experiments to determine which CNS cell types are most vulnerable to 5-FU revealed clinically relevant concentrations of this agent to be particularly toxic to in vitro populations of neural progenitor cells and oligodendrocytes. These cell populations were also extremely vulnerable to 5 -FU in vivo. Short-term systemic administration of $5-\mathrm{FU}$ to mice caused marked short-term increases in apoptosis and more-prolonged reductions in cell division in the multiple CNS regions examined. This acute damage was accompanied by a syndrome of worsening delayed damage to myelin. This 'late' damage was associated with abnormal transcriptional regulation in oligodendrocytes and extensive myelin pathology. Previous studies have shown that loss of myelin can affect the speed of impulse conduction. Analysis of auditory function in 5-FU-treated mice revealed progressive latency of impulse transduction over time-a finding that may facilitate the noninvasive analysis of chemotherapy-induced myelin damage. The authors conclude that their animal model of late chemotherapyinduced damage to the CNS should facilitate further research into the neurological effects of chemotherapeutic agents.

Original article Han R et al. (2008) Systemic 5-fluoruracil treatment causes a syndrome of delayed myelin destruction in the central nervous system. J Biol 7: 12

\section{Tarenflurbil slows decline in patients with mild Alzheimer disease}

Deposition of amyloid $\beta(A \beta)$ protein in the brain is thought to be instigated by the 42-amino-acid variant of the peptide, $A \beta_{42}$. Prolonged treatment 